\section{P01.08 STRUCTURAL AND CONTEXTUAL FACTORS IN THE SEXUAL HEALTH OF ADOLESCENT ABORIGINAL AUSTRALIANS: A SYSTEMATIC REVIEW}

Catherine MacPhail* ${ }^{*}$ Kathy McKay. School of Health, University of New England, Armidale, NSW, Australia

\subsection{6/sextrans-2015-052270.218}

Background Levels of STIs and teenage pregnancy among Aboriginal and Torres Strait Islander populations are significantly higher than among non-Indigenous populations. However, recent research has shown few differences in the sexual behaviour of young Indigenous and non-Indigenous Australians. We conducted a systematic review to examine the multi-dimensional and socially constructed nature of adolescent sexuality with a view to developing meaningful STI and pregnancy reduction programmes for young Indigenous Australians.

Methods Following standard guidelines for systematic reviews we searched eight relevant databases for published studies. We focused on publications since January 2003 using search terms: adolescent, youth, sexual, Australia, Indigenous, Aboriginal and Torres Strait. Given the limited amount of literature available we included all study designs and studies of broader age ranges or populations, but where Indigenous and adolescent populations were disaggregated in study results.

Results We identified an initial 2,718 citations through the database search. After removing duplicates and citations that were not peer-reviewed or research articles, we screened 2,266 articles and excluded 2,158. The remaining 180 articles were reviewed in full: 93 did not meet inclusion criteria. Our review finally comprised 15 research papers, representing eleven distinct studies.

Across the literature included in the review a number of key social determinants of poor sexual health were identified. These included alcohol, poverty, gender inequalities, intergenerational trauma, limited access to education and employment, shame, constrained adult role models and aspirations and restricted access to (sexual) health services. We examine the role of these factors with regard to adolescent Indigenous sexual health.

Conclusions International research argues that promoting good sexual health requires a broader focus on sexuality. The sociocultural and historical contexts of Indigenous communities suggest that focusing on strengths-based approaches to sexual health with peer or community facilitation might be acceptable and effective for young Indigenous Australians.

Disclosure of interest statement Catherine MacPhail and Kathy McKay were supported by the Collaborative Research Network on Mental Health and Wellbeing in Rural Communities, Department of Industry, Innovation, Science, Research and Tertiary Education, Commonwealth Government of Australia.

\section{P01.09 TRENDS IN SELECTED MEASURES OF RACIAL AND ETHNIC DISPARITIES IN GONORRHOEA AND SYPHILIS IN THE UNITED STATES, 1981-2012}

CG Patel, HW Chesson, TL Gift*, SO Aral. Centers for Disease Control and Prevention

10.1136/sextrans-2015-052270.219

Introduction The purpose of this study was to examine different measures of racial and ethnic disparities in the reported cases of primary and secondary (P\&S) syphilis and gonorrhoea from 1981 to 2012 in the United States.
Methods For each year from 1981 to 2012, we calculated values for five disparity measures: the Gini coefficient, two versions of the index of disparity (unweighted and weighted by population subgroup size), the population attributable fraction, and the black-to-white rate ratio. We also examined annual changes in these measures. Specifically, we examined whether or not the five measures yielded consistent assessments as to whether racial and ethnic disparity was increasing or decreasing from one year to the next.

Results Overall, the disparity measures we examined were generally consistent with one another. However, from any given year to the next, the various disparity measures could yield divergent results in terms of whether racial/ethnic disparities in STDs are increasing or decreasing as well as in terms of the relative magnitude of the change. Over the 31 -year period, $\geq 4$ of the 5 index measures agreed on the direction of change 28 times for both P\&S syphilis and gonorrhoea. The most common measure that had a different direction of change from the other measures was the population attributable fraction.

Conclusion Our findings illustrated two well-known limitations of relative measures of racial/ethnic disparities in health. First, relative measures of disparity can decrease due to increases in STD incidence rates among Non-Hispanic Whites (or increase due to decreases in STD incidence among Non-Hispanic Whites). Second, whether or not racial and ethnic disparities increased from one year to the next can differ across measures of disparity. Reliance on just one measure of disparity can at times lead to divergent conclusions about changes in disparities over short time periods.

Disclosure of interest statement The findings and conclusions in this study are those of the authors and do not necessarily represent the official position of the United States Centres for Disease Control and Prevention (CDC). No external funding was received for this study and the authors have no conflicts of interest.

\section{P01.10 HIV/STI INFECTIONS AND RISK BEHAVIOUR AMONG DAO ETHNIC PEOPLE IN 2006 AND 2012 IN VIET NAM}

TD Quang* ${ }^{*}$ DC Thanh, LA Tuan, NTT Ha, NA Tuan. National Institute of Hygiene and Epidemiology, Ha Noi, Viet Nam

\subsection{6/sextrans-2015-052270.220}

Introduction Due to cultural characteristics, there are some potential risks for HIV/STI transmission including early sexual debut and multiple sex partners among Dao ethnic people. This study aims to measure HIV/STI prevalence and risk behaviour among this ethnic minority in Viet Nam.

Methods Household survey was carried out to recruit 807 and 802 Dao people in 2006 and 2012. A two-stage cluster sampling design was used. The households were chosen randomly based on the list of local authority. All women and men aged 15-49 was eligible to be selected into the survey. Participants were asked to be interviewed and provide $3 \mathrm{ml}$ bloods for HIV/STI testing.

Results Syphilis prevalence was $3.4 \%$ and $5.6 \%$ in 2006 and 2012, respectively. HIV prevalence was $0.0 \%$ in both rounds. Mean age of sexual debut was 17.5 (SD: 2.3 years old) and 17 years old (SD: 1.7 years old) in 2006 and 2012, respectively. Correct knowledge on HIV prevention was $18.7 \%$ and $50.2 \%$ in 2006 and 2012, respectively. Having multiple sex partners in the last 12 months was $5.8 \%, 8.6 \%$ for men and $3.0 \%$ for women, 
and $30.1 \%, 38.5 \%$ for men and $22.2 \%$ for women, in 2006 and 2012 respectively. Always using condom with any sexual partners in the last 12 months was $3.5 \%$ and $5.1 \%$ in 2006 and 2012, respectively. Using drug was $1.4 \%$ in both rounds. HIV testing and knowing the result were $1.1 \%$ and $31.8 \%$ in 2006 and 2012, respectively. Among aged 15-24 groups, the correct knowledge on HIV prevention was increased from 21.0\% (2006) to $52.9 \%$ (2012); always using condom with any sexual partners in the last 12 months was increased from 5.9\% (2006) to $12.0 \%$ (2012) and syphilis prevalence was decreased from $3.1 \%$ (2006) to $1.6 \%$ (2012).

Conclusion High syphilis prevalence combined with high sexual risk behaviour such as multiple sex partners and low consistent condom use suggests high potential risk for HIV/STI transmission among Dao ethnic people. In addition, low-level knowledge on HIV/AIDS prevention and STI treatment warrants extra attention. HIV prevention effort needs to ensure reaching this remote and vulnerable ethnicity with focus on young in Viet Nam.

Disclosure of interest None.

\section{P01.11 UNDERSTANDING THE GREATER BURDEN OF STIS AMONG BLACK CARIBBEANS IN THE UK: EVIDENCE FROM A SYSTEMATIC REVIEW}

${ }^{1} \mathrm{~S}$ Wayal*, ${ }^{1} \mathrm{C}$ Griffiths, ${ }^{1} \mathrm{C}$ Mercer, ${ }^{1} \mathrm{M}$ Gerrussu, ${ }^{2} \mathrm{G}$ Hughes. ${ }^{1} \mathrm{C}$ entre for Sexual Health and HIV Research, University College London, UK; ${ }^{2}$ Public Health England, Colindale, UK

\subsection{6/sextrans-2015-052270.221}

Background In the UK, Black Caribbeans are disproportionately affected by STIs. We conducted a systematic review of attitudinal, behavioural and contextual risk factors of this inequality.

Methods Ten electronic databases were searched for studies on risk factors and drivers of STI among UK Black Caribbeans from 1948 to 30/11/2014. Two independent reviewers screened all identified abstracts and extracted data from selected studies using standardised forms.

Results Of 3220 abstracts identified, 165 were included in the review. STI risk among Black Caribbeans is higher compared to other ethnic groups and varies by gender and age. Being single and reporting first intercourse aged $<16,>1$ new sex partner in the past year, concurrency, and assortative sexual mixing were identified as risk factors. STIs were considered of lower priority than HIV/unplanned pregnancy. Barriers to condom use, especially among women with older and regular partners, were reported. Compared to other ethnic groups, Black Caribbeans were more likely to have ever attended a STI clinic and tested for HIV, but Black Caribbean women were more likely to report delays in seeking care and be sexually active whilst symptomatic. Perceived negative attitudes of clinic staff of the same ethnicity towards young women negatively affected care-seeking.

Conclusion Sexual behavioural risk factors or access to care did not fully explain the disproportionate STIs burden among Black Caribbeans highlighting the need for further evidence on contextual drivers of STIs. STI reduction interventions should be gender-specific, informed by partnership patterns and address attitudes to STIs and sexual health care-seeking.

Disclosure of interest statement Nothing to declare.

\section{P02 - New media in STI prevention}

\section{P02.01 SYSTEMATIC REVIEW AND META-ANALYSIS OF RANDOMISED CONTROL TRIALS OF INTERACTIVE DIGITAL INTERVENTIONS FOR SEXUAL HEALTH PROMOTION}

${ }^{1} \mathrm{~S}$ Wayal*, ${ }^{1} \mathrm{JV}$ Bailey, ${ }^{1} \mathrm{E}$ Murray, ${ }^{1} \mathrm{G}$ Rait, ${ }^{1} \mathrm{RW}$ Morris, ${ }^{2} \mathrm{R}$ Peacock, ${ }^{1}$ I Nazareth. ${ }^{1}$ University College London, UK; ${ }^{2}$ Middlexex University, London, UK

\subsection{6/sextrans-2015-052270.222}

Background Sexual ill-health is a global concern. Digital technology offers enormous potential for health promotion. This systematic review assessed effectiveness of interactive digital interventions (IDI) for sexual health promotion.

Methods IDI are interactive programmes providing information, decision support, behaviour-change support and/or emotional support. We searched 40 electronic databases for randomised controlled trials (RCT) of IDI for sexual health promotion. Cochrane Collaboration methods were used to determine the effectiveness of IDI vs. minimal interventions (e.g. waiting list) (comparison1); face-to-face interventions (comparison 2); and different designs of IDI (comparison 3). Separate meta-analyses were conducted for comparisons 1,2 , and 3 , by type of outcome (knowledge, self-efficacy, intention, sexual behaviour and biological outcomes). Results were pooled using a random effects model to calculate standardised mean differences (SMDs) and odds ratios (ORs). Subgroup analyses tested the following prespecified factors: age, risk grouping, and settings (online, healthcare, educational).

Results We identified 34 RCTs (10,758 participants). Comparison 1: IDI had beneficial effect on knowledge (SMD 0.43, 95\% CI 0.14 to 0.71 ); safer sex self-efficacy (SMD 0.11, 95\% CI 0.03 to 0.18 ) and intention (SMD $0.13,95 \%$ CI 0.05 to 0.22 ). There was no effect on sexual behaviour (OR 1.15, 95\% CI 0.97 to 1.36 ) or biological outcomes (OR $0.81,95 \%$ CI 0.56 to 1.16). Comparison 2: IDI improved knowledge (SMD 0.36, 95\% CI 0.1 to 0.58 ), and intention (SMD $0.46,95 \%$ CI 0.06 to 0.85 ), but not self-efficacy (SMD $0.38,95 \%$ CI 0.01 to 0.77 ). Comparison 3: Tailoring showed a beneficial effect on sexual behaviour (OR 2.64, 95\% CI 1.45 to 4.80). No subgroup differences were noted. No data were available for cost-effectiveness. Conclusions IDIs can effectively enhance knowledge, self-efficacy, intention, and tailored IDIs can improve sexual behaviour. Further evidence is needed to understand how to translate these positive effects of IDIs into improved sexual health, and how IDIs work.

Disclosure of interest Nothing to Declare.

\section{P02.02 ANOTHER DIGI-GAP! INTEGRATING MULTIFACETED TRIAGING APPROACHES TO ASSIST AND SPEED TECHNOLOGICAL TRANSITION TO ELECTRONIC MEDICAL DOCUMENTATION AND BOOKING SYSTEMS}

${ }^{1}$ MM Florance*, ${ }^{1}$ A Cheshire, ${ }^{1}$ B Hadlow, ${ }^{1,2}$ A Ubrihien. ${ }^{1}$ North Shore Sexual Health Service, Northern Sydney LHD, Sydney, Australia; ${ }^{2}$ HIV and Related Programs Unit, Western Sydney Sexual Health Centre, Western Sydney Local Health District

10.1136/sextrans-2015-052270.223 